\title{
Characterization of cardiopulmonary interactions and explor- ing their prognostic value in acute bronchiolitis: A prospective cardiopulmonary ultrasound study
}

\author{
Moises Rodriguez-Gonzalez ${ }^{1}$, Patricia Rodriguez-Campoy ${ }^{2}$, Lorena Estepa-Pedregosa ${ }^{2}$, Ana Estalella-Mendoza ${ }^{2}$, \\ Ana Castellano-Martinez ${ }^{3 *}$, Jose Carlos Flores-Gonzalez ${ }^{2}$ \\ 1 Pediatric Cardiology Division. Puerta del Mar University Hospital. Cadiz Spain. \\ 2 Pediatric Intensive Care Unit. Puerta del Mar University Hospital. Cadiz Spain. \\ 3 Pediatric Nephrology Division. Puerta del Mar University Hospital. Cadiz Spain. \\ * Correspondence: anacastellanomart@gmail.com.
}

\begin{abstract}
We aimed to delineate cardiopulmonary interactions in acute bronchiolitis and to evaluate the capacity of a combined cardiopulmonary ultrasonography to predict the need for respiratory support. This was a prospective observational single-center study that includes infants $<12$ month of age admitted to a hospital due to acute bronchiolitis. All the included patients underwent clinical, laboratory and cardiopulmonary ultrasonographic evaluation at the same time point within 24 hours of hospital admission. The existence of significant correlation between cardiac and respiratory parameters was the primary outcome. The association of different cardiopulmonary variables with the need of respiratory support higher than $\mathrm{O} 2$, the length of stay hospitalization, the PICU stay, and the duration of respiratory support were a secondary outcome. We enrolled 112 infants (median age $1(0.5-3)$ months; $62 \%$ males) hospitalized with acute bronchiolitis. Increased values of the pulmonary variables (BROSJOD score, pCO2 and LUS) showed moderate correlations with NTproBNP and all echocardiographic parameters indicative of pulmonary hypertension and myocardial dysfunction. Up to $36(32 \%)$ infants required respiratory support during the hospitalization. This group presented with higher lung ultrasound score $(p<0.001)$, and increased values of Tei index $(\mathrm{p}<0.001)$ and pulmonary artery pressures $(\mathrm{p}<0.001)$. All the analyzed respiratory and cardiac variables showed moderate to strong correlations with the LOS hospitalization and the time of respiratory support. Lung ultrasound and echocardiography showed a moderate to strong predictive accuracy for the need of respiratory support in the ROC analysis, with AUC varying from 0.74 to 0.87 . Conclusion: Those cases of bronchiolitis with a worse pulmonary status presented with a more impaired cardiac status. Cardiopulmonary ultrasonography could be a good strategy to easily identify high-risk population for a complicated acute bronchiolitis hospitalization.
\end{abstract}

Keywords: Acute bronchiolitis; Lung ultrasound; Echocardiography; Point of care ultrasonography; Cardiopulmonary ultrasound; Cardiopulmonary interactions; Pulmonary hypertension; Myocardial strain; NT-proBNP

\section{Introduction}

Bronchiolitis is the viral lower respiratory infection that most often affects children under 2 years of age, with a peak of incidence and morbidity in young infants (under 3 months of age) [1,2]. It is characterized by small-airway inflammation, edema, mucus production, and necrosis, leading to obstruction of distal bronchioles and pulmonary air trapping with subsequent acute impairment in gas exchange between lungs and blood resulting in hypoxia with or without hypercapnia, and secondary acidosis. Of note, approximately $5 \%-$ $10 \%$ of infants with acute bronchiolitis develop acute respiratory failure (ARF) requiring respiratory support and PICU admission [1,2]. 
The existence of a physiological interdependence between the cardiovascular and respiratory systems has long been recognized. The heart and lungs are anatomically coupled through the pulmonary circulation and coexist within the sealed thoracic cavity, making the function of these systems highly interdependent. During ARF these interactions are magnified, with a striking effect on pulmonary vasculature and myocardial function $[3,4]$. An understanding of the interplay between the cardiac and respiratory systems could be important in the management of severe bronchiolitis. However, the presence and the clinical consequences of adverse cardiopulmonary interactions (CPI) in common respiratory diseases, such as bronchiolitis, remain incompletely understood and poorly studied.

Clinical scores have proven to be inaccurate in predicting prognosis in acute bronchiolitis, with weak interobserver reliability and limited construct validity [5]. Thus, lung ultrasound (LUS) is now emerging as a valuable tool in assessment of respiratory status in bronchiolitis. Literature supports the use of LUS as a reliable imaging test that could benefit the clinical management of bronchiolitis [6-8]. The presence of myocardial strain assessed by echocardiography is also being increasingly recognized in this setting, where approximately $20 \%$ of patients present myocardial dysfunction (MD) or pulmonary hypertension (PHT) at the time of hospitalization [9]. The integrative cardiopulmonary ultrasound (CPU) approach is an emerging non-invasive technique that combines lung ultrasound (LU) and echocardiography to evaluate lung and cardiac conditions simultaneously, especially in the critical care setting $[10,11]$. To date, there are few investigations evaluating concomitant dysfunction of the pulmonary and cardiac systems in critically ill children [12], and no publications in infants with acute bronchiolitis. Using bedside CPU would allow for a rapid and thorough assessment of both the cardiovascular and respiratory state in these setting. We hypothesized that patients with more severe respiratory status will present with higher rates of pulmonary hypertension and myocardial dysfunction. Although these findings could be theoretically plausible, there is still scarce evidence documenting CPI and their prognostic implications in this setting, and there are no publications using CPU as a noninvasive tool for prognosis assessment in bronchiolitis.

The primary objective of this study was to explore the influence of the respiratory state over the heart and pulmonary circulation using CPU in infants with bronchiolitis at early stages of the disease. A secondary objective was to evaluate the prognostic implications of adverse CPI and the predictive values of different CPU measurements in this setting.

\section{Materials and Methods}

\subsection{Design, setting and study population}

We conducted a single center, prospective and observational study from October 2018 to March 2020 at the Pediatric Department of a tertiary University hospital in Spain. We included infants hospitalized with acute bronchiolitis. The diagnosis and management were made by the pediatrician on charge following current international recommendations [13]. Exclusion criteria were chronic lung diseases, congenital heart diseases, immunodeficiency, previous cardiopulmonary resuscitation, detection of bacterial superinfection, initiation of respiratory support or administration of epinephrine or intravenous fluid before the research intervention, incomplete research intervention or medical records, and poor-quality ultrasound images. The local Ethics Committee approved the protocol and informed parental consent was obtained from all patients.

\subsection{Research intervention}

All the included patients underwent clinical, laboratory and cardiopulmonary ultrasonographic evaluation at the same time point within 24 hours of hospital admission. 
PCR analysis of nasopharyngeal mucus for the respiratory syncytial virus was routinely analyzed in all patients during hospitalization.

\subsubsection{Pulmonary status assessment}

The respiratory state was clinically measured using the bronchiolitis of Sant Joan de Déu (BROSJOD) score [14]. This score includes five parameters: heart rate, respiratory rate, $\mathrm{SpO} 2$, pulmonary auscultation, and costal retractions. It ranges from 0 to 14 points. A score greater than 10 points is defined as severe bronchiolitis. Venous $\mathrm{pH}$ and $\mathrm{pCO} 2$ levels were used as laboratory markers of the respiratory state. A significant hypercarbia was defined as pCO2 $>55 \mathrm{cmH} 20$ independently of pH levels. Lung ultrasound (LU) was performed by 3 intensive care pediatricians with at least 2 years of experience in LU in routine practice according to the recommendations for point of care lung ultrasound by Volpicelli et al [15]. Sonographic examinations were obtained in supine position in calmed patients without need of sedation during the procedure. A Doppler ultrasound machine (SonoScape S8) equipped with a high-frequency (16-4 MHz) linear transducer was used to explore 3 chest projections in each hemithorax (Figure 1). We did not evaluate posterior/paravertebral projections due to the difficulty in performing them in these patients, which makes them not very feasible in clinical practice, and furthermore, we believe that these would only provide limited information, since the posterior fields are the ones, most frequently affected in the initial phases of acute bronchiolitis, probably due to the supine position of these patients. However, the involvement of the anterior lung fields may reflect greater severity and worse evolution, as shown by the study by Bueno-Campaña et al [16]. Each area was longitudinally explored in a cephalocaudal direction evaluating each intercostal space. In each area, the presence of B-lines and subpleural consolidations was analyzed using a lung ultrasound score (LUS) (Figure $2 \& 3$ ). A higher LUS means a more severe pulmonary involvement. A LUS $>10$ was considered a moderate to severe pulmonary disease in this study. Pleural line abnormalities were not analyzed, as they add little value to consolidations and interstitial syndrome with respect to the assessment of acute bronchiolitis severity and prognosis [16, 17].
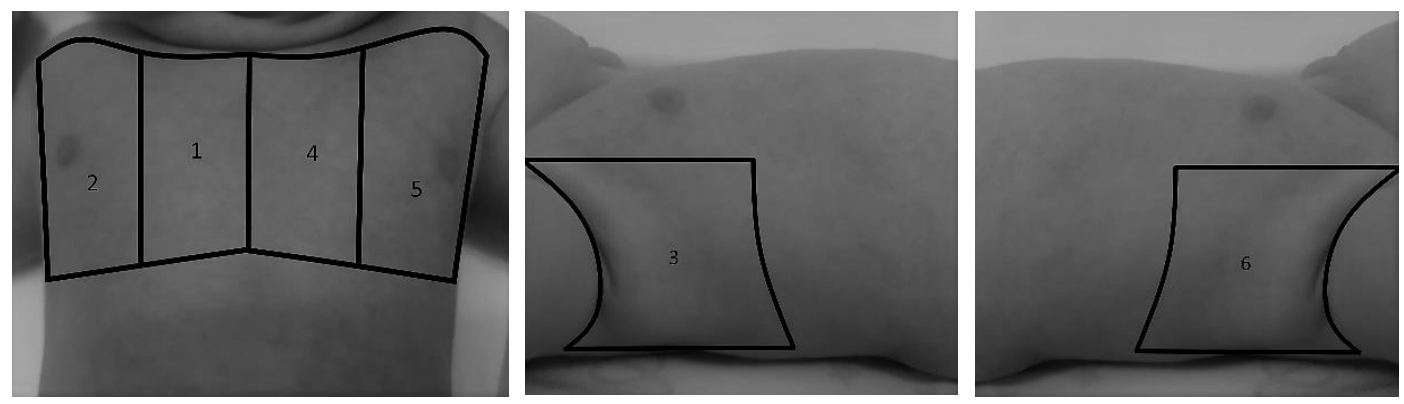

Figure 1. Chest projections used in our study protocol. 1: right anteromedial (from parasternal line to right mid-clavicular line); 2: right anterolateral (from right mid-clavicular line to right anterior axillary line); 3: right lateral (from right anterior axillary line to right posterior axillary line); 4: left anteromedial (from parasternal line to left mid-clavicular line); 5: left anterolateral (from left mid-clavicular line to left anterior axillary line); 6: left lateral (from left anterior axillary line to left posterior axillary line). 


\begin{tabular}{|c|c|c|c|c|c|c|}
\hline \multicolumn{9}{|c|}{ LU Score (LUS) } \\
\hline \multicolumn{2}{|c|}{ B-lines } & $\mathbf{2}$ & $\mathbf{3}$ & $\mathbf{0}$ & $\mathbf{1}$ \\
\hline $\mathbf{0}$ & $\mathbf{1}$ & $\begin{array}{c}\text { Consolidations } \\
\text { tercostal space } \\
\text { or } \\
\text { lines }\end{array}$ & $\begin{array}{c}\geq 2 \text { intercostal spaces } \\
\text { whith adjacent white } \\
\text { white lung in an } \\
\text { intercostal space }\end{array}$ & $\begin{array}{c}\text { lung } \\
\text { Consolidation }\end{array}$ & $\begin{array}{c}\text { No } \\
\text { consolidations }\end{array}$ & $\begin{array}{c}\text { consolidation }<1 \\
\mathrm{~cm}\end{array}$ \\
$\begin{array}{c}\text { Consolidation } \geq 1 \\
\mathrm{~cm} \\
\text { or multiple con- } \\
\text { solidations }\end{array}$ \\
\hline
\end{tabular}

Figure 2. Lung Ultrasound Score (LUS): based on number of B-lines, extension of B-lines and the presence of subpleural consolidation. A score from 0 to 5 was given for each projection in each hemithorax for a maximum LUS of 30 points.

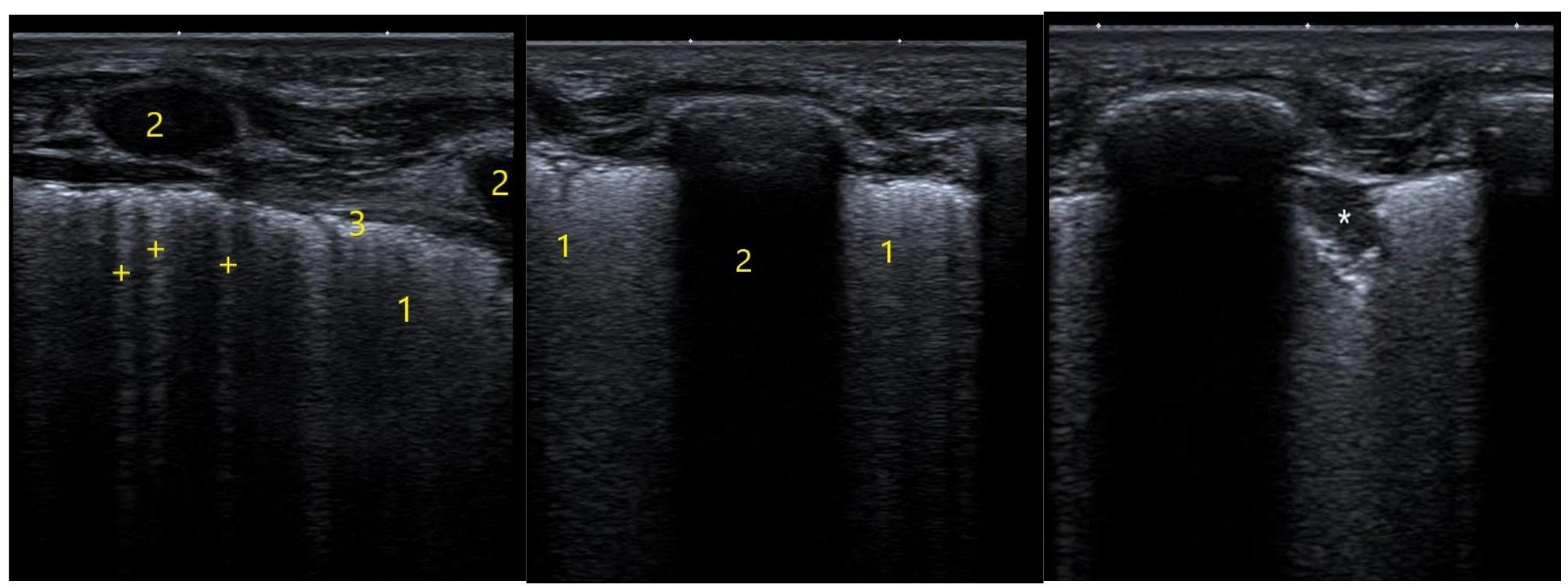

Figure 3. This is a lung ultrasound image showing: A. Left side panel: B lines. In this image multiple B lines (+) are observed in the intercostal space, some coalescing, giving rise to a white lung image. Irregular and poorly defined pleuropulmonary line. 1: coalescing b lines, white lung; 2: ribs; 3: pleuropulmonary line. B. Central panel: Coalescing b lines or white lung (1) in twoadjacent intercostal spaces delimited by a rib (2). C. Right side panel: Subpleural consolidation. Hypoechoic image with frayed edges $\left(^{*}\right)$.

\subsubsection{Cardiac status assessment}

NT-proBNP was used as a laboratory parameter of myocardial strain. Serum NTproBNP levels were determined within 2 hours of venous blood samples collection using the electrochemical luminescence immunoassay Elecsys proBNP II (Roche Diagnostics; $5 \mathrm{pg} / \mathrm{ml}$ of limit of detection, $1.5 \%$ intra-run variation; less than $3 \%$ inter-run variation). Serum levels plus than $1121 \mathrm{pg} / \mathrm{ml}$ (99th percentile for 1-12 months of age) were considered elevated [18].

Standard techniques to obtain M-mode, two-dimensional and Doppler (colour, pulsed, continuous and tissue Doppler) echocardiograms were performed by the same experienced paediatric cardiologist as recommended in the guidelines for the paediatric echocardiography [19-23]. Images were obtained using a Phillips IE33 ultrasound scanner with an 8 or $12-\mathrm{MHz}$ sectorial transducer. Each examination was recorded, and all the studies were reviewed off-line by 1 observer who was blinded to the patient's clinical profile. All echocardiographic measurements represent the average of 5 beats. Echocardiographic parameters used for cardiac function and pulmonary pressures evaluation

included: Left ventricle (LV) ejection fraction (LVEF), tricuspid annular plane systolic excursion (TAPSE), LV and RV Tei index (LVTX and RVTX) (Figure 3), systolic gradient 
of the tricuspid regurgitation jet (TRJG), RV outflow tract acceleration time to ejection time ratio (ATET), LV systolic eccentricity index (LVEI), and RV to LV ratio (RVLV) (Ta

ble1 \& Figure 4).

Table 1. Echocardiographic parameters used to evaluate myocardial involvement in our population.

\begin{tabular}{cccc}
\hline Echo parameter & Cardiac parameter evaluated & Abnormal value & Significance \\
\hline LVEF & LV systolic function & $<50 \%$ & Myocardial dysfunction \\
TAPSE & RV systolic function & $<2$ SD for BS & Myocardial dysfunction \\
LVTX & LV global (systolic and diastolic) function & $>0.50$ & Myocardial dysfunction \\
RVTX & RV global (systolic and diastolic) function & $>0.50$ & Myocardial dysfunction \\
TRJG & Pulmonary pressures & $>40 \mathrm{mmHg}$ & Pulmonary Hypertension \\
ATET & Pulmonary pressures & $<0.29$ & Pulmonary Hypertension \\
LVEI & RV systolic pressure & $>1.3$ & Pulmonary Hypertension \\
RVLV & RV dimension & $>0.6$ & Dilated RV \\
\hline
\end{tabular}

Legend: LVEF: left ventricle ejection fraction; TAPSE: tricuspid annular plane systolic excursion; LVTX: left ventricular Tei index; RVTX: right ventricular Tei index; TRJG: tricuspid regurgitation jet gradient; ATET: acceleration time/ejection time ratio; LVEI: left ventricular eccentricity index; RV/LV: right ventricular/left ventricular ratio 


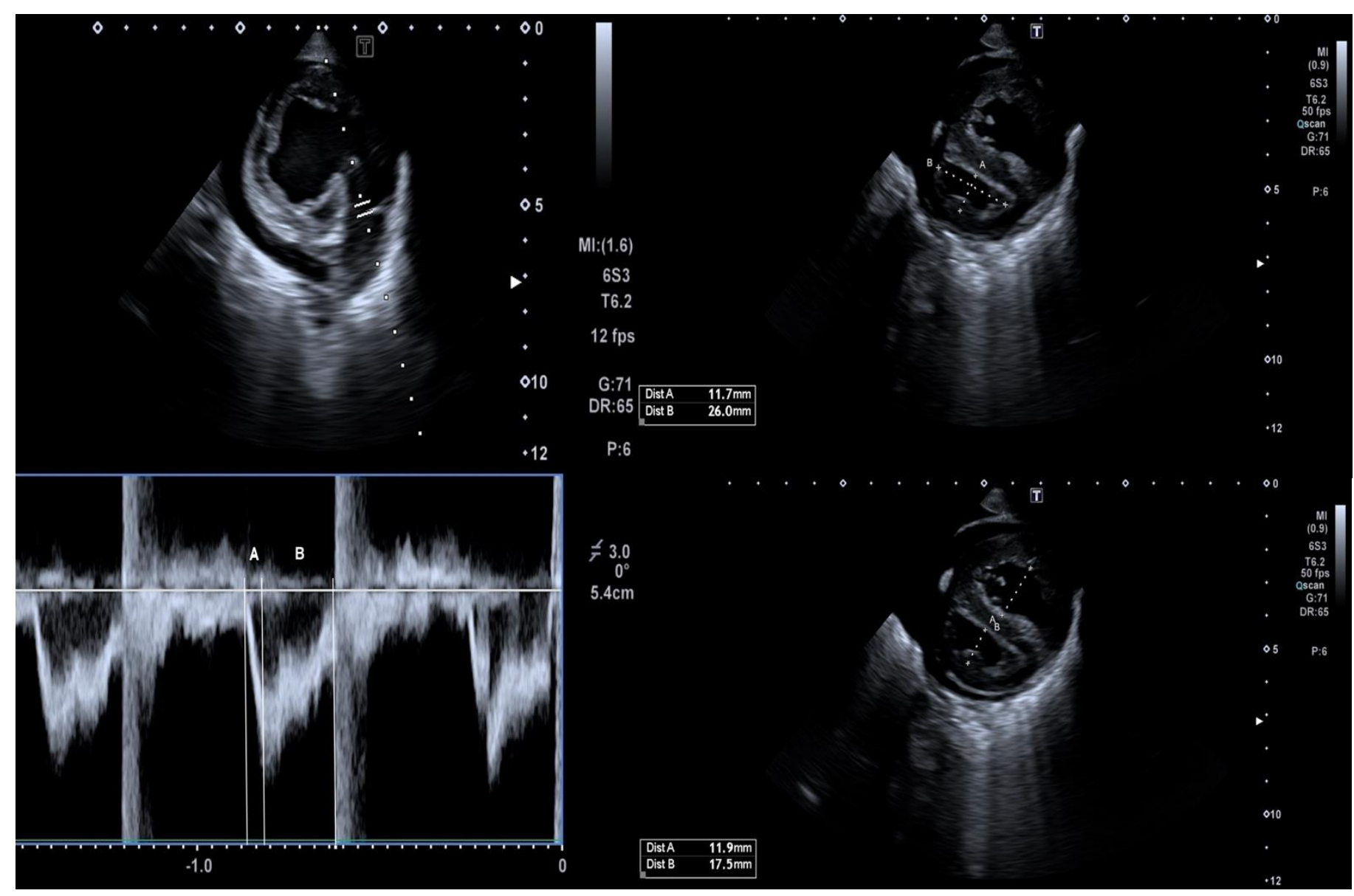

Figure 4. This figure shows the semiquantitative echocardiographic parameters used to assess pulmonary artery pressures in this study. In a short axis view we interrogated the RV outflow tract flow with Pulsed wave Doppler (Left sided image) and calculated from resultant spectral Doppler imaging the ATET ratio (A/B). We also calculated the systolic LVEI at the level of the papillary muscles (right-up sided image) as the ratio between mediolateral and anterposterior diammeters of the LV (A/B). Finally, we calculated the RVLV ratio at the same level (B/A).

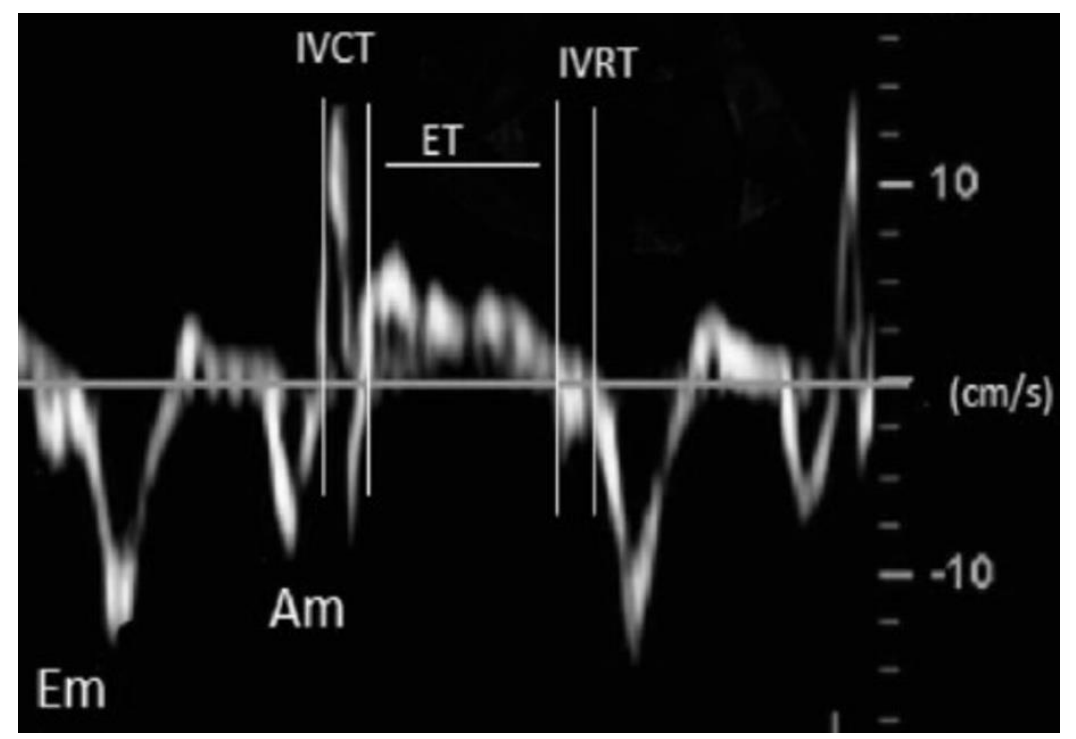

Figure 5. This figure shows a Doppler tissue imaging obtained at the mitral annulus for the calculation of the TEI index as a parameter for estimation of LV function in this study. IVCT (isovolumetric contraction time); IVRT (isovolumetric relaxation time); ET (ejection time). 
The follow-up started at the research intervention time and finished at hospital discharge. At the end of the study period an independent observer collected participants data through our institution's electronic medical records and included in a relational database. Baseline information included demographic, clinical, laboratory and ultrasound data.

Outcome variables included the need of PICU admission, PICU stay, type and duration of respiratory support: oxygen $(\mathrm{O} 2)$, high-flow oxygen (HFO), noninvasive ventilation (NIV), or mechanical ventilation (MV); and LOS hospitalization. The existence of significant correlation between cardiac and respiratory parameters was the primary outcome in this investigation. The association of different cardiopulmonary variables with the need of respiratory support higher than $\mathrm{O} 2$, the length of stay hospitalization, the PICU stay, and the duration of respiratory support were a secondary outcome of this study.

\subsection{Statistics}

Continuous data are presented as median (range) or mean (standard deviation) after testing normality with the Shapiro-Wilk test. Categorical data are presented as frequencies and percentage. Mean and median comparisons were performed using Student's t-test or Wilcoxon Mann-Whitney test as appropriate. Proportions were compared using the Chi-square test or exact methods as necessary. Spearman's coefficient test was used to verify correlation between variables. Logistic regression and receiver operator curve (ROC) analysis were used to explore the predictive accuracy of each marker for respiratory support. Odds ratio (OR), sensitivity, specificity, and predictive values with 95\% confidence interval were estimated for the cut-off value at which they are considered abnormal. A significance level of 0.05 was used for all statistical tests. Statistical analyses were performed with the use of Stata 16.0 (Stata Corp., College Station, Texas).

\section{Results}

\subsection{Baseline characteristics}

We enrolled 112 infants (median age 1 (0.5-3) months; 62\% males) hospitalized with acute bronchiolitis. Table 2 summarizes the baseline characteristics and clinical variables related to clinical evolution. Table 3 shows the prevalence of abnormal values of respiratory and cardiac parameters evaluated in our population.

\subsection{Cardiopulmonary interactions in bronchiolitis}

Increased values of the pulmonary variables (BROSJOD score, pCO2 and LUS) showed moderate correlations with NT-proBNP and all echocardiographic parameters indicative of pulmonary hypertension (TRJG, ATET, LVEI, and RVLV) and global myocardial dysfunction (LVTX and RVTX), but not with parameters of systolic myocardial dysfunction (LVEF or TAPSE) (Table 4).

\subsection{Relationship of cardiac and respiratory variables with a severe course of the disease.}

Of the 112 (\%) participants, up to 36 (32\%) required respiratory support during the hospitalization (Table 5). Regarding the respiratory evaluation, the respiratory support group presented with higher BROSJOD score ( $\mathrm{p}<0.001), \mathrm{pCO} 2(<0.001)$, LUS $(\mathrm{p}<0.001)$, and lower $\mathrm{pH}$ levels $(\mathrm{p}<0.001)$. With respect to the cardiac assessment, this group showed higher levels of NT-proBNP $(p<0.001)$, increased values of LVTX $(p<0.001)$, RVTX $(p<0.001)$, TRJG $(p<0.001)$, LVEI $(p<0.001)$, RVLV $(p<0.001)$, and lower values of ATET $(p<0.001)$.All the analyzed respiratory variables and all cardiac variables, except to LVEF and TAPSE, showed moderate to strong correlations with the LOS hospitalization and the time of respiratory support. None of the studied variables analyzed presented association with the PICU stay (Table 6). All the cardiac and respiratory variables explored, except to LVEF and TAPSE, were associated with increased risk for the need of respiratory support 
and also showed a moderate to strong predictive accuracy for the need of respiratory support in the ROC analysis, with AUC varying from 0.74 to 0.87 (Table 7).

Table 2. Demographic and outcome data of the study population

\section{Patient number 112}

Age (months)*

Weight $(\mathrm{kg})^{*}$

Gender (male)^

Time from onset of symptoms to ED evaluation (days)*

RSV positive ${ }^{\wedge}$

Nebulizations^

Oxygen therapy (nasal canulae) ${ }^{\wedge}$

Duration (days)*

$\mathrm{HFNC}^{\wedge}$

Duration (days)*

PICU admission^

PICU stay (days)*
Results

$1(0.5-3)$

$4.6(3.7-5.8)$

62 (55)

$1(0-2)$

86 (77)

$63(58)$

$56(50)$

$2(1-4)$

$3(2.5)$

$1(1-2)$

$36(32)$

$6(4-9)$ 
$\wedge$ Data presented in frequency and percentage. * Data presented in median and interquartile range. ED: emergency department. BROSJOD: Bronchiolitis Score of Sant Joan de Déu; RSV: respiratory syncytial virus; HFNC: high flow nasal cannula; PICU: pediatric intensive care unit; NIV: noninvasive ventilation; MV: mechanical ventilation; LOS: length of stay.

Table 3. Prevalence of abnormal or severe respiratory and cardiac parameters evaluated in this cohort.

\begin{tabular}{|c|c|}
\hline Patient number (112) & N (\%) \\
\hline \multicolumn{2}{|c|}{ Respiratory variables } \\
\hline BROSJOD score $>10$ points $^{\wedge}$ & $24(21)$ \\
\hline $\mathrm{pH}<7.30$ & $30(26)$ \\
\hline $\mathrm{pCO} 2>55 \mathrm{cmH} 20$ & $51(45)$ \\
\hline LUS > 10 points $\wedge$ & $25(22)$ \\
\hline \multicolumn{2}{|c|}{ Cardiac variables } \\
\hline NT-proBNP > $1121 \mathrm{pg} / \mathrm{ml}^{*}$ & $57(51)$ \\
\hline \multicolumn{2}{|l|}{ Myocardial function } \\
\hline LVEF $<50 \%$ & $0(0)$ \\
\hline TAPSE $<2$ Zscore for BSA & $4(3.5)$ \\
\hline LVTX $>0.5$ & $26(23)$ \\
\hline RVTX $>0.5$ & $25(22)$ \\
\hline
\end{tabular}


Legend: BROSJOD: Bronchiolitis Score of Sant Joan de Déu; LUS: lung ultrasound score; NTproBNP: N-Terminal Pro Brain Natriuretic Peptide; LVEF: left ventricle ejection fraction; TAPSE: tricuspid annular plane systolic excursion; LVTX: left ventricular Tei index; RVTX: right ventricular Tei index; TRJG: tricuspid regurgitation jet gradient; ATET: acceleration time/ejection time ratio; LVEI: left ventricular eccentricity index; RV/LV: right ventricular/left ventricular ratio

Table 4. Correlation of the cardiac variables with the respiratory variables studied in this investigation.

\begin{tabular}{|c|c|c|c|c|c|c|}
\hline \multirow{2}{*}{ Cardiac variables } & \multicolumn{2}{|c|}{ BROSJOD score } & \multicolumn{2}{|c|}{$\mathrm{pCO} 2$} & \multicolumn{2}{|c|}{ LUS score } \\
\hline & $\rho$ & P-Value & $\rho$ & P-value & $\rho$ & P-value \\
\hline NT-proBNP $(\mathrm{pg} / \mathrm{mL}){ }^{*}$ & 0.37 & 0.001 & 0.48 & 0.000 & 0.46 & 0.000 \\
\hline $\operatorname{LVEF}(\%)^{* *}$ & 0.01 & Ns. & -0.05 & Ns. & -0.05 & Ns. \\
\hline $\operatorname{LVTX}^{* *}$ & 0.25 & 0.006 & 0.39 & 0.000 & 0.33 & 0.000 \\
\hline TAPSE $^{* *}$ & -0.10 & Ns. & -0.18 & Ns. & -0.19 & Ns. \\
\hline $\mathrm{RVTX}^{* *}$ & 0.35 & 0.000 & 0.44 & 0.000 & 0.37 & 0.000 \\
\hline TRJG $(\mathrm{mmHg})^{* *}, \mathrm{n} 50$ & 0.36 & 0.010 & 0.56 & 0.000 & 0.34 & 0.014 \\
\hline $\mathrm{ATET}^{* *}$ & -0.37 & 0.001 & -0.43 & 0.000 & -0.34 & 0.000 \\
\hline LVEI $^{* *}$ & 0.38 & 0.000 & 0.48 & 0.000 & 0.45 & 0.000 \\
\hline RVLV $^{* *}$ & 0.26 & 0.005 & 0.30 & 0.002 & 0.32 & 0.000 \\
\hline
\end{tabular}

n: sample size. CC: Spearman's or Pearson correlation coefficient. BROSJOD: Bronchiolitis Score of Sant Joan de Déu; LUS: lung ultrasound score.; NT-proBNP: N-Terminal Pro Brain Natriuretic Peptide;LVEF: left ventricle ejection fraction; LVTX: left ventricular Tei index;; TAPSE: tricuspid annular plane systolic excursion; RVTX: right ventricular Tei index; TRJG: tricuspid regurgitation jet gradient; ATET: acceleration time/ejection time ratio; LVEI: left ventricular eccentricity index; RV/LV: right ventricular/left ventricular ratio. Ns. ( $p>0.05)$ 
Table 5. Respiratory and cardiological evaluation according to the need of respiratory support during hospitalization.

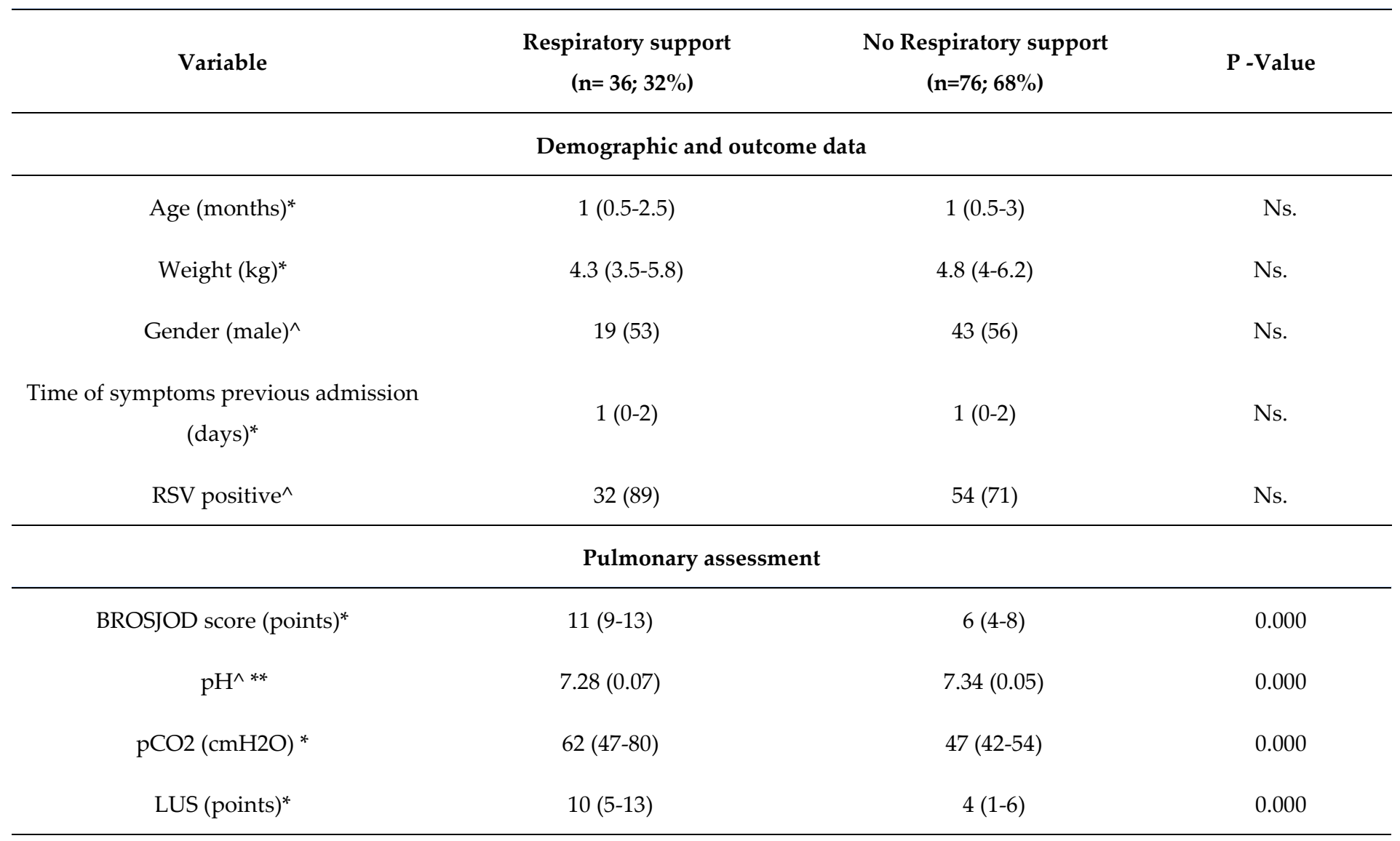

\section{Cardiac assessment}




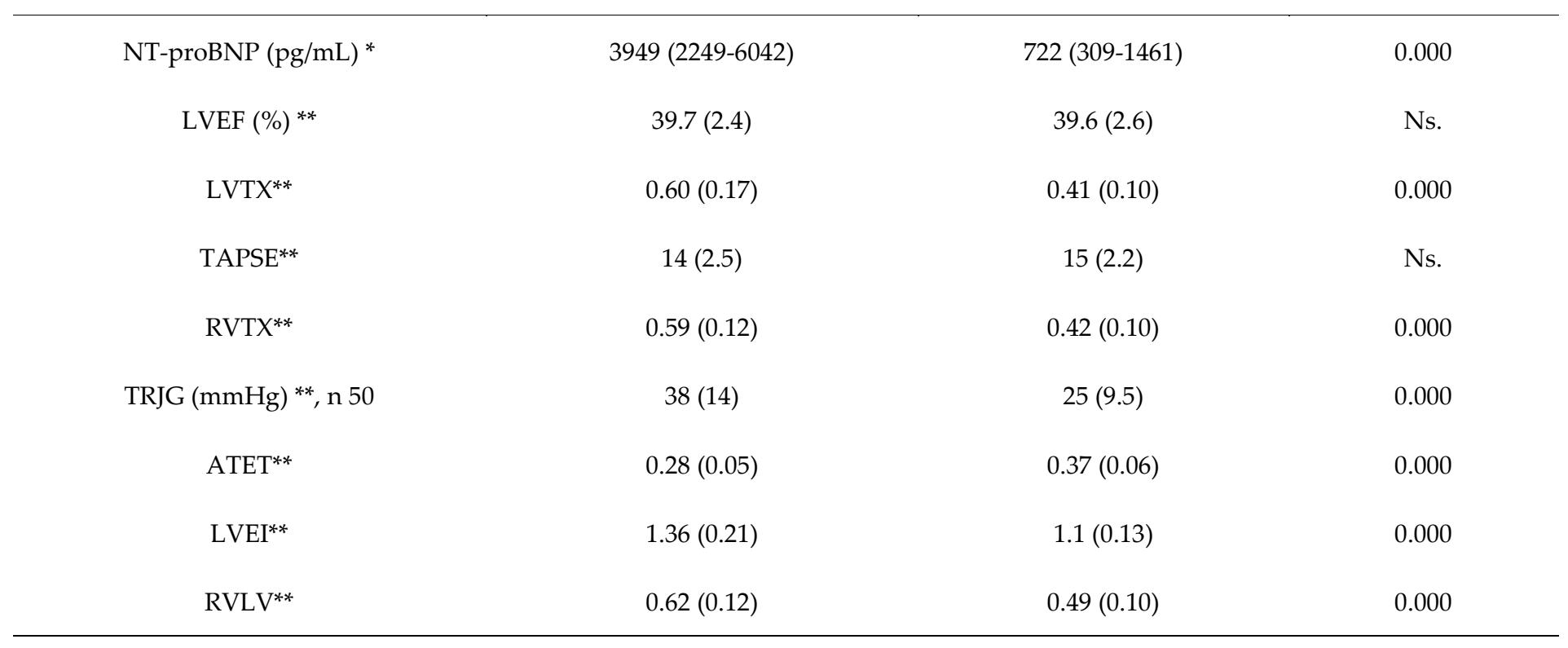

\footnotetext{
$\wedge$ Data presented in frequency and percentage. ${ }^{*}$ Data presented in median and interquartile range. ${ }^{* *}$ Data presented in mean (standard deviation). Ns (p 0.05). RSV: respiratory syncytial virus. BROSJOD: Bronchiolitis Score of Sant Joan de Déu; LUS: lung ultrasound score; NT-proBNP: N-Terminal Pro Brain Natriuretic Peptide;LVEF: left ventricle ejection fraction; LVTX: left ventricular Tei index;; TAPSE: tricuspid annular plane systolic excursion; RVTX: right ventricular Tei index;; TRJG: tricuspid regurgitation jet gradient; ATET: acceleration time/ejection time ratio; LVEI: left ventricular eccentricity index; RV/LV: right ventricular/left ventricular ratio.
}

Table 6. Correlation of cardiac and respiratory variables with outcome data.

\begin{tabular}{|c|c|c|c|c|c|c|}
\hline \multirow{2}{*}{$\begin{array}{l}\text { Cardiac and } \\
\text { respiratory variables }\end{array}$} & \multicolumn{2}{|c|}{ LOS Hospitalization } & \multicolumn{2}{|c|}{ PICU stay } & \multicolumn{2}{|c|}{ Duration of RS } \\
\hline & $\rho$ & P-Value & $\rho$ & P-value & $\rho$ & $\mathrm{P}$-value \\
\hline BROSJOD score & 0.55 & $<0.001$ & -0.08 & Ns. & 0.47 & $<0.001$ \\
\hline $\mathrm{pH}$ & -0.34 & $<0.001$ & -0.21 & Ns. & -0.23 & 0.021 \\
\hline $\mathrm{pCO} 2$ & 0.42 & $<0.001$ & 0.03 & Ns. & 0.29 & 0.004 \\
\hline LUS & 0.58 & $<0.001$ & 0.06 & Ns. & 0.02 & Ns. \\
\hline NT-proBNP & 0.60 & $<0.001$ & 0.14 & Ns. & 0.50 & $<0.001$ \\
\hline LVEF & 0.01 & Ns. & -0.05 & Ns. & -0.05 & Ns. \\
\hline LVTX & 0.49 & $<0.001$ & 0.17 & Ns. & 0.40 & $<0.001$ \\
\hline TAPSE & -0.10 & Ns. & -0.18 & Ns. & -0.19 & Ns. \\
\hline RVTX & 0.58 & $<0.001$ & 0.23 & Ns. & 0.42 & $<0.001$ \\
\hline TRJG, n 50 & 0.48 & $<0.001$ & -0.13 & Ns. & 0.31 & 0.024 \\
\hline ATET & -0.52 & $<0.001$ & -0.18 & Ns. & -0.45 & $<0.001$ \\
\hline LVEI & 0.58 & $<0.001$ & 0.20 & Ns. & 0.44 & $<0.001$ \\
\hline RVLV & 0.54 & $<0.001$ & 0.21 & Ns. & 0.38 & $<0.001$ \\
\hline
\end{tabular}


n: sample size. CC: Spearman's or Pearson correlation coefficient. LOS: length of stay; PICU: pediatric intensive critical care unit; RS: respiratory support;. BROSJOD: Bronchiolitis Score of Sant Joan de Déu; LUS: lung ultrasound score; NT-proBNP: N-Terminal Pro Brain Natriuretic Peptide; LVEF: left ventricle ejection fraction; LVTX: left ventricular Tei index; TAPSE: tricuspid annular plane systolic excursion; RVTX: right ventricular Tei index; ATET: acceleration time/ejection time ratio; LVEI: left ventricular eccentricity index; RV/LV: right ventricular/left ventricular ratio. Ns. (p>0.05).

Table 7. Predictive value of different cardiopulmonary parameters for the need of advanced respiratory support.

\begin{tabular}{cccccccc}
\hline Predictive variables & OR (CI95\%) & AUC (CI95\%) & S & Sp & PPV & NPV \\
\hline BROSJOD $>10$ points & $28(12-125)$ & $0.87(0.78-0.93)$ & 0.91 & 0.61 & 0.91 & 0.76 \\
pH $<7.30$ & $3.5(1.5-8.5)$ & $0.72(0.61-0.83)$ & 0.44 & 0.81 & 0.53 & 0.75 \\
pCO2 $>55$ cmH2O & $4.3(2-10)$ & $0.74(0.62-0.86)$ & 0.69 & 0.65 & 0.49 & 0.82 \\
LUS $>10$ points & $6(2.3-15)$ & $0.77(0.67-0.85)$ & 0.44 & 0.88 & 0.64 & 0.77 \\
NT-proBNP $>$ 1121 pg/mL & $9(3.3-24)$ & $0.83(0.73-0.91)$ & 0.85 & 0.71 & 0.55 & 0.90 \\
LVTX $>0.5$ & $8.5(3-22)$ & $0.80(0.69-0.89)$ & 0.50 & 0.89 & 0.70 & 0.79 \\
RVTX $>0.5$ & $18(5.8-54)$ & $0.84(0.76-0.92)$ & 0.55 & 0.93 & 0.80 & 0.81 \\
LVEI $>1.3$ & $15(5.5-40)$ & $0.84(0.75-0.92)$ & 0.64 & 0.89 & 0.74 & 0.84 \\
ATET $<0.29$ & $7.4(3-18)$ & $0.83(0.75-0.91)$ & 0.55 & 0.85 & 0.65 & 0.80 \\
RVLV $>0.6$ & $8.4(3.3-21)$ & $0.77(0.68-0.86)$ & 0.61 & 0.84 & 0.64 & 0.82 \\
\hline
\end{tabular}

AUC: Area under the receiver operator curve. S: sensitivity. Sp: specificity. PPV: positive predictive value. NPV: negative predictive value. BROSJOD: Bronchiolitis Score of Sant Joan de Déu; LUS: lung ultrasound score; NT-proBNP: N-Terminal Pro Brain Natriuretic Peptide;; LVTX: left ventricular Tei index; RVTX: right ventricular Tei index; LVEI: left ventricular eccentricity index; ATET: acceleration time/ejection time ratio; RV/LV: right ventricular/left ventricular ratio

\section{Discussion}

In this prospective study we employed an integrative cardiopulmonary approach to comprehensively assess the pulmonary and cardiac condition of previously healthy infants with acute bronchiolitis at the time of hospitalization. Under normal conditions, CPI are inconsequential; however, they may become exaggerated and of great importance in certain acute pulmonary diseases. Through simultaneous clinical, laboratory, and CPU assessment we shown that a more severe respiratory state with hypoxia, respiratory acidosis and increased respiratory effort, resulted in increased rates of raised pulmonary pressures 
and declined myocardial dysfunction, even in absence of underlying heart disease. Of note, the presence of adverse CPI at early stages of the disease were associated with a severe course of the disease, with higher rates of respiratory support, PICU admission and prolonged hospitalizations. Therefore, the early recognition of cardiopulmonary impairment could be crucial to identify high risk population for a complicated acute bronchiolitis.

\subsection{Pulmonary status in acute bronchiolitis}

The viral induced inflammatory response causes peri bronchial inflammatory infiltrate, interstitial edema, and epithelial desquamation, leading to small-airway obstruction, air trapping, and compromise of the lung parenchyma (consolidations and atelectasis). Almost all studies in $\mathrm{AB}$ describe elevated inspiratory and expiratory resistance, reduced tidal volume, increased auto-PEEP, lung hyperinflation, increased FRC, and, finally, decreased respiratory compliance $[24,25]$. Clinically, these lung conditions manifest in most severe cases as increased respiratory effort and impaired gas exchange with subsequent hypoxemia and acidosis. As we showed in this study, LU in infants with bronchiolitis can show diffuse and heterogeneous alveolar-interstitial involvement, which can range from mild alterations, such as pleuropulmonary line abnormalities and B-line pattern, to large pulmonary consolidations $[6-8,12,16]$. Most studies about LU in bronchiolitis used an own score as we did. Evaluating children with this LUS up to $25 \%$ of patients hospitalized with bronchiolitis presented a moderate to severe pulmonary impairment. This high rate of patients with pulmonary alterations reinforces the need of LU screening early after admission. According previously reported, we observed a good correlation between clinical and ultrasound findings $[12,16]$. Of note, LU can identify lung abnormalities not revealed by chest-X-ray [17]. Given the short time needed to get a LU report, this technique could become the routine imaging modality for assessment of pulmonary status in infants with bronchiolitis.

\subsection{Cardiac status in acute bronchiolitis}

Although these infants are usually admitted to the hospital for management of respiratory distress and hypoxia, the relevance of the cardiovascular involvement in these patients is increasingly documented. Cardiovascular complications are described in up to $9 \%$ of hospitalized patients [26]. Significant cardiac output and stroke volume changes have been demonstrated in infants without heart disease who were hospitalized for bronchiolitis in relation with their respiratory distress [27]. Up to $50 \%$ of patients have a positive troponin or NT-proBNP assay at the time of hospitalization. In addition, infants with bronchiolitis admitted to intensive care with hemodynamic instability and acute respiratory failure are associated with increased levels of cardiac biomarkers (cardiac troponin and NT-proBNP) compared with mild cases [28-32]. A recent meta-analysis showed the presence of pulmonary hypertension (pooled incidence of $20 \%(95 \% \mathrm{CI}, 11-30 \%)$ and myocardial dysfunction (pooled incidence of 5\% (95\% CI, 1-9\%) in 395 previously healthy infants with acute bronchiolitis [9]. Interestingly, myocardial dysfunction is frequently biventricular, as demonstrated by strain rate echocardiography (STE) in the cohort of Massolo et al (32\% of infants with BA) [33]. Consistent with previous evidence, approximately $20 \%-30 \%$ of our patients developed PH and subsequent MD; and 50\% presented raised NT-proBNP levels. Also, the presence of echocardiographic alterations correlated well with clinical score, $\mathrm{pH}$ and pCO2. These results remark the potential utility of early point of care echocardiographic evaluation in infants hospitalized with acute bronchiolitis as a non-invasive tool to assess severity and guide management in a similar way that LU.

To detect myocardial dysfunction in BA, echocardiographic study by tissue Doppler imaging (TDI) and speckle tackle echocardiography has proven to be a more sensitive method than conventional echocardiography. Tissue Doppler and STE imaging has provided more load-independent methods to assess myocardial function with the additional 
advantage that high quality two-dimensional images are no longer essential for reproducible recordings. Consistently, we observed that the TDI-derived Tei Index, was significantly impaired in those patients with a more compromised respiratory state. In addition, a combined systolic and diastolic measurement such as Tei index, may be more reflective of overall myocardial dysfunction than those conventional methods for assessing only systolic function. Regarding estimation of pulmonary pressures, we did not detect any difference in pulmonary arterial pressure based on standard TRJG findings between groups of severity. TRJG jet can be difficult to be detected and alternative semiquantitative parameters could be useful in this setting. Remarkably, the ATET, LVEI, and RVLV could be measured in all infants unlike tricuspid regurgitation, which was detectable in approximately a half of them in our study. As previously reported, the inclusion of these parameters in the echocardiographic evaluation of these patients could improve the detection of PHT in this setting [9].

\subsection{Cardiopulmonary interactions in acute bronchiolitis}

Our major finding was that echocardiographic parameters assessing myocardial function and pulmonary pressures and the biomarker of myocardial strain NT-proBNP showed a moderate correlation with the respiratory clinical score that includes the respiratory rate and hypoxemia, with respiratory acidosis, and with our LUS. Therefore, our study documented those factors such as lung edema, consolidations and airway obstruction leading to hypoxia, respiratory acidosis and increased respiratory effort, causes a deleterious effect in the cardiac performance in bronchiolitis. Of note, this is the first clinical study to apply a homogeneous prospective methodology investigating cardiopulmonary interactions by a combined and integrative cardiopulmonary approach in this clinically relevant setting.

The most likely underlying mechanism explaining our findings might be an increase in pulmonary vascular resistances and right heart after load and subsequent reduced right and left ventricular functions $[29,30]$. Pulmonary vasoconstriction can be induced by several factors in acute bronchiolitis including respiratory acidosis, hypoxemia, release of vasoactive inflammatory mediators, direct inflammatory endothelial damage, direct compression of pulmonary blood vessels secondary to the reduction in functional lung volume and the increased alveolar pressure, and a vascular remodeling phenomenon. Recent basic science studies in mouse models by Kimura et al., shows that there are structural changes in pulmonary vasculature in $\mathrm{AB}$, and that suppression of tumorigenicity 2 factor (ST2) signaling is involved in the development of RSV-associated PH providing histopathological and pathophysiological substrate for this hypothesis [34,35].

With the increased pulmonary vascular resistance, RV structure and function will be impaired by the increased after load and because of ventricular interdependence, can also cause LV myocardial dysfunction due to an abnormal pattern of relaxation and contraction secondary to geometric changes occurring in the LV [36]. In addition, the reduction in pulmonary flow generated by RV dysfunction leads to a reduced LV preload, which is followed by a reduce stroke volume ejected by LV [37]. Furthermore, the exaggerated respiratory effort in bronchiolitis, can increase the importance of these interactions. The dominant effect of a spontaneous inspiratory effort is an increased filling of the right heart and diastolic ventricular interaction with reduced LV preload and combined increased LV after load $[3,4,38]$. The increase in the work of breathing also activates the sympathetic nervous system and renin-angiotensin-aldosterone systems, contributing to increases in ventricular after load, altogether contributing to a decrease in LV output [3,4,38]. Also, oxygen consumption is increased to values over $50 \%$ of total oxygen consumption due to the higher activity of the diaphragm and respiratory muscles during respiratory distress $[3,4,38]$. Altogether, $\mathrm{MD}$ and reduced pulmonary and systemic $\mathrm{CO}$ with increased oxygen consumption could explain the sepsis-like or myocarditis-like appearance of some infants with severe $\mathrm{AB}$. 


\subsection{Predictive accuracy of cardiopulmonary ultrasound for adverse outcomes in acute bronchiolitis}

LU is increasingly used to assess the respiratory state and even to predict evolution and guide treatment in bronchiolitis with promising results $[8,12]$. In the study by Bueno-Campaña et al. the ultrasound lung score combined with the WDF scale and age shows a moderate prognostic accuracy for the need of respiratory support, with an AUC of 0.84 (95\%CI: 0.781-0.909) and, both posterior consolidations $>1 \mathrm{~cm}$ and greater extension of the interstitial pattern in anterior fields, present a higher correlation with the need of respiratory support [16]. Consistently, we found that the group of patients requiring respiratory support had a worse LUS and the 10-point LUS showed a moderate prognostic accuracy for the need for respiratory support (AUC 0.78. 95\% CI 0.685-0.873) in bronchiolitis. The differences regarding accuracy could be explained by the different characteristic of the populations studied and the different LUS employed.

Echocardiography is emerging as another useful tool to assess severity in AB. The use of echocardiography was first applied to assess the cardiac state in children with underlying heart disease [39]. However, in recent years there is evidence supporting the presence of myocardial dysfunction and pulmonary hypertension also in previously healthy infants. Of note, cardiac involvement is related with the severity and prognosis of $\mathrm{AB}$ (pooled relative risk $=16 ; 95 \% \mathrm{CI}, 8.2-31.5$ ) [9]. In our cohort, DTI-derived Echo analysis reveals increased pulmonary pressures and reduced myocardial function in relation with more impaired respiratory status. Beyond the predictive capacity provided by clinical and pulmonary ultrasound, most variables analyzed showed a moderate or good predictive value for the need of respiratory support at the cut-off value at which they are abnormal in our ROC analysis. Notably, conventional echocardiographic parameters for myocardial function and pulmonary hypertension such as TAPSE, LVEF and TRJG were not useful to predict the need of PICU in this study. As LVEF and TAPSE are dependent of the ventricular geometry, preload and afterload, myocardial dysfunction could not be properly reflected by them in the acute respiratory setting, where the exaggerated respiratory efforts induce significant differences in lung volume leading to marked changes in left and right ventricular loading conditions. Regarding parameters for detection of PHT, the ATET is a good surrogate marker for pulmonary artery pressure. ATET $<0.30$ has been validated against RHC to have $97 \%$ sensitivity and $95 \%$ specificity at detecting pulmonary hypertension in children [40]. In our cohort the ATET showed a good predictive accuracy for the need of respiratory support with an AUC of 0.83 (95\%CI: 0.75-0.91). Therefore, ATET could be a rapid and accurate parameter for PHT in acute bronchiolitis.

Both echocardiography and LU have been well described as having utility for guiding the diagnosis and management of the critically ill patient $[10,41]$. Both techniques can be performed rapidly, safely, and repeatedly at point of care by the pediatrician on charge of the case. We observed that the presence of adverse CPI at early stages of the disease were associated with a severe course of the disease, with higher rates of respiratory support, PICU admission and prolonged hospitalizations. As there is no parameter with perfect predictive capacity in $\mathrm{AB}$ our results support the use combined $\mathrm{CPU}$ as a non-invasive tool to improve the early identification of patients that will require PICU admission or respiratory support. Notably, previous studies showed that CPU has obvious advantages in investigating the etiology of acute respiratory failure in the pediatric ED setting [42]. Most unknown severe cardiac conditions in infants can mimic the presentation of $\mathrm{AB}$. Hence, infants with respiratory insufficiency from bronchiolitis may have similar clinical features to infants with congestive heart failure, presenting with irritability, fever, tachypnea, tachycardia and a mottled appearance. Therefore, we believe that a CPU approach will have broad application prospects in the initial evaluation of $A B$.

\section{Limitations}


This was a single-center study with relatively few patients, so for stronger conclusions a larger study is needed. Also, it may not be possible to extrapolate our findings to clinical practice in other hospitals. CPU is high operator dependent, and this is an inherent limitation. Echocardiographic measures were not analyzed for intra-reader or inter-reader variability, although all parameters analyzed were already described to have high interreader and intra-reader agreement in previous studies. There is no validated LUS in bronchiolitis, and the classification of the ultrasound findings employed in the study was developed specifically for using in this study. Another limitation of our study was the lack of gold standard diagnostic tests for myocardial dysfunction and PHT, mainly cardiac magnetic resonance imaging and right heart catheterism.

\section{Conclusions}

Our study documented that pulmonary and cardiac function are closely connected in children hospitalized with bronchiolitis. Those patients with a worse pulmonary status presented with a more impaired cardiac status. As those patients with adverse CPI developed a more severe bronchiolitis, the current findings demonstrate that CPU examination could be a good strategy with an added value to easily identify high-risk population for a complicated AB hospitalization. Rather than compartmentalizing the two techniques, we favor integration of echocardiography with LU in this setting.

Author Contributions: Conceptualization, M.RG. and J.C.FG.; methodology, M.RG. and J.C.FG.; software, M. RG.; validation, P.RC and L.EP; formal analysis, M.RG..; investigation, A.CM., A.EM., P.RC and L.EP; resources, M.RG. and J.C.FG.; data curation, X.X.; writing-original draft preparation, M.RG and A.CM.; writing - review and editing, J.C.FG, P.RC , L.EP A.CM., and A.EM.; visualization, X.X.; supervision, M.RG. and J.C.FG.; project administration, J.C.FG.; funding acquisition, J.C.FG. All authors have read and agreed to the published version of the manuscript

Funding: This research received no external funding

Institutional Review Board Statement: The study was conducted according to the guidelines of the Declaration of Helsinki and approved by the Institutional Review Board (or Ethics Committee) of Puerta del Mar University Hospital (date of approval: 25 $5^{\text {th }}$ October 2018)." OR "Ethical review and approval were waived for this study, due to REASON (please provide a detailed justification)." OR "Not applicable." for studies not involving humans or animals. You might also choose to exclude this statement if the study did not involve humans or animals.

Informed Consent Statement: Informed consent was obtained from all subjects involved in the study.

Data Availability Statement: The data presented in this study are available within this article.

Conflicts of Interest: The authors declare no conflict of interest.

\section{References}

1. Bozzola E, Ciarlitto C, Guolo S, Brusco C, Cerone G, Antilici L, Schettini L, Piscitelli AL, Chiara Vittucci A, Cutrera R, Raponi M, Villani A. Respiratory Syncytial Virus Bronchiolitis in Infancy: The Acute Hospitalization Cost. Front Pediatr. 2021 Jan 18;8:594898. doi: 10.3389/fped.2020.594898. PMID: 33537260; PMCID: PMC7848214.

2. Schlapbach L, Straney L, Gelbart B, Alexander J, Franklin D, Beca J, Whitty JA, Ganu S, Wilkins B, Slater A, Croston E, Erickson S, Schibler A; Australian \& New Zealand Intensive Care Society (ANZICS) Centre for Outcomes \& Resource Evaluation (CORE) and the Australian \& New Zealand Intensive Care Society (ANZICS) Paediatric Study Group. Burden of disease and change in practice in critically ill infants with bronchiolitis. Eur Respir J. 2017 Jun 1;49(6):1601648. doi: 10.1183/13993003.01648-2016. PMID: 28572120.

3. Pinsky MR. Cardiopulmonary Interactions: Physiologic Basis and Clinical Applications. Ann Am Thorac Soc. 2018 Feb;15(Suppl 1):S45-S48. doi: 10.1513/AnnalsATS.201704-339FR. PMID: 28820609; PMCID: PMC5822394.

4. Bronicki RA. Cardiopulmonary Interactions in Children with Heart Failure. Curr Cardiol Rev. 2016;12(2):104-6. doi: 10.2174/1573403x12666151119164802. PMID: 26585038; PMCID: PMC4861937. 
5. Siraj S, Stark W, McKinley SD, Morrison JM, Sochet AA. The bronchiolitis severity score: An assessment of face validity, construct validity, and interobserver reliability. Pediatr Pulmonol. 2021 Jun;56(6):1739-1744. doi: 10.1002/ppul.25337. Epub 2021 Mar 8. PMID: 33629813.

6. Basile V, Di Mauro A, Scalini E, Comes P, Lofù I, Mostert M, Tafuri S, Manzionna MM. Lung ultrasound: a useful tool in diagnosis and management of bronchiolitis. BMC Pediatr. 2015 May 21;15:63. doi: 10.1186/s12887-015-0380-1. PMID: 25993984; PMCID: PMC4494717.

7. La Regina DP, Bloise S, Pepino D, lovine E, Laudisa M, Cristiani L, Nicolai A, Nenna R, Mancino E, Di Mattia G, Petrarca L, Matera L, Frassanito A, Midulla F. Lung ultrasound in bronchiolitis. Pediatr Pulmonol. 2021 Jan;56(1):234-239. doi: 10.1002/ppul.25156. Epub 2020 Nov 24. PMID: 33151023.

8. Di Mauro A, Ammirabile A, Quercia M, Panza R, Capozza M, Manzionna MM, Laforgia N. Acute Bronchiolitis: Is There a Role for Lung Ultrasound? Diagnostics (Basel). 2019 Nov 1;9(4):172. doi: 10.3390/diagnostics9040172. PMID: 31683953; PMCID: PMC6963954.

9. Rodriguez-Gonzalez M, Perez-Reviriego AA, Castellano-Martinez A, Cascales-Poyatos HM. The Assessment of Myocardial Strain by Cardiac Imaging in Healthy Infants with Acute Bronchiolitis: A Systematic Review and MetaAnalysis. Diagnostics (Basel). 2020 Jun 8;10(6):382. doi: 10.3390/diagnostics10060382. PMID: 32521769; PMCID: PMC7345904.

10. Le Coz J, Orlandini S, Titomanlio L, Rinaldi VE. Point of care ultrasonography in the pediatric emergency department. Ital J Pediatr. 2018 Jul 27;44(1):87. doi: 10.1186/s13052-018-0520-y. PMID: 30053886; PMCID: PMC6064059.

11. Lichtenstein D, Karakitsos D. Integrating lung ultrasound in the hemodynamic evaluation of acute circulatory failure (the fluid administration limited by lung sonography protocol). J Crit Care. 2012 Oct;27(5):533.e11-9. doi: 10.1016/j.jcrc.2012.03.004. Epub 2012 Apr 18. PMID: 22520492.

12. Buonsenso D, De Rose C, Ferro V, Morello R, Musolino A, Valentini P. Lung ultrasound to detect cardiopulmonary interactions in acutely ill children. Pediatr Pulmonol. 2021 Nov 11. doi: 10.1002/ppul.25755. Epub ahead of print. PMID: 34761881.

13. Ralston SL, Lieberthal AS, Meissner HC, Alverson BK, Baley JE, Gadomski AM, Johnson DW, Light MJ, Maraqa NF, Mendonca EA, Phelan KJ, Zorc JJ, Stanko-Lopp D, Brown MA, Nathanson I, Rosenblum E, Sayles S 3rd, HernandezCancio S; American Academy of Pediatrics. Clinical practice guideline: the diagnosis, management, and prevention of bronchiolitis. Pediatrics. 2014 Nov;134(5):e1474-502. doi: 10.1542/peds.2014-2742. Erratum in: Pediatrics. 2015 Oct;136(4):782. PMID: 25349312.

14. Balaguer M, Alejandre C, Vila D, Esteban E, Carrasco JL, Cambra FJ, Jordan I. Bronchiolitis Score of Sant Joan de Déu: BROSJOD Score, validation and usefulness. Pediatr Pulmonol. 2017 Apr;52(4):533-539. doi: 10.1002/ppul.23546. Epub 2016 Nov 7. PMID: 28328090.

15. Volpicelli G, Elbarbary M, Blaivas M, Lichtenstein DA, Mathis G, Kirkpatrick AW, Melniker L, Gargani L, Noble VE, Via G, Dean A, Tsung JW, Soldati G, Copetti R, Bouhemad B, Reissig A, Agricola E, Rouby JJ, Arbelot C, Liteplo A, Sargsyan A, Silva F, Hoppmann R, Breitkreutz R, Seibel A, Neri L, Storti E, Petrovic T; International Liaison Committee on Lung Ultrasound (ILC-LUS) for International Consensus Conference on Lung Ultrasound (ICC-LUS). International evidence-based recommendations for point-of-care lung ultrasound. Intensive Care Med. 2012 Apr;38(4):577-91. doi: 10.1007/s00134-012-2513-4. Epub 2012 Mar 6. PMID: 22392031.

16. Bueno-Campaña M, Sainz T, Alba M, Del Rosal T, Mendez-Echevarría A, Echevarria R, Tagarro A, Ruperez-Lucas M, Herreros ML, Latorre L, Calvo C. Lung ultrasound for prediction of respiratory support in infants with acute bronchiolitis: A cohort study. Pediatr Pulmonol. 2019 Jun;54(6):873-880. doi: 10.1002/ppul.24287. Epub 2019 Mar 5. PMID: 30838805. 
17. Caiulo VA, Gargani L, Caiulo S, Fisicaro A, Moramarco F, Latini G, Picano E. Lung ultrasound in bronchiolitis: comparison with chest X-ray. Eur J Pediatr. 2011 Nov;170(11):1427-33. doi: 10.1007/s00431-011-1461-2. Epub 2011 Apr 6. PMID: 21468639.

18. Nir A, Lindinger A, Rauh M, Bar-Oz B, Laer S, Schwachtgen L, Koch A, Falkenberg J, Mir TS. NT-pro-B-type natriuretic peptide in infants and children: reference values based on combined data from four studies. Pediatr Cardiol. 2009 Jan;30(1):3-8. doi: 10.1007/s00246-008-9258-4. Epub 2008 Jul 4. PMID: 18600369.

19. Lai WW, Geva T, Shirali GS, Frommelt PC, Humes RA, Brook MM, Pignatelli RH, Rychik J; Task Force of the Pediatric Council of the American Society of Echocardiography; Pediatric Council of the American Society of Echocardiography. Guidelines and standards for performance of a pediatric echocardiogram: a report from the Task Force of the Pediatric Council of the American Society of Echocardiography. J Am Soc Echocardiogr. 2006 Dec;19(12):1413-30. doi: 10.1016/j.echo.2006.09.001. PMID: 17138024.

20. Koestenberger M, Apitz C, Abdul-Khaliq H, Hansmann G. Transthoracic echocardiography for the evaluation of children and adolescents with suspected or confirmed pulmonary hypertension. Expert consensus statement on the diagnosis and treatment of paediatric pulmonary hypertension. The European Paediatric Pulmonary Vascular Disease Network, endorsed by ISHLT and D6PK. Heart. 2016 May;102 Suppl 2:ii14-22. doi: 10.1136/heartjnl-2014307200. PMID: 27053693.

21. Koestenberger M, Ravekes W, Everett AD, Stueger HP, Heinzl B, Gamillscheg A, Cvirn G, Boysen A, Fandl A, Nagel B. Right ventricular function in infants, children and adolescents: reference values of the tricuspid annular plane systolic excursion (TAPSE) in 640 healthy patients and calculation of z score values. J Am Soc Echocardiogr. 2009 Jun;22(6):715-9. doi: 10.1016/j.echo.2009.03.026. Epub 2009 May 7. PMID: 19423286.

22. Cui W, Roberson DA. Left ventricular Tei index in children: comparison of tissue Doppler imaging, pulsed wave Doppler, and M-mode echocardiography normal values. J Am Soc Echocardiogr. 2006 Dec;19(12):1438-45. doi: 10.1016/j.echo.2006.06.006. PMID: 17138026.

23. Abraham S, Weismann CG. Left Ventricular End-Systolic Eccentricity Index for Assessment of Pulmonary Hypertension in Infants. Echocardiography. 2016 Jun;33(6):910-5. doi: 10.1111/echo.13171. Epub 2016 Jan 16. PMID: 26773570.

24. Rao X, Liu X, Jiang Q, Jiao A, Jiang Y. [Pulmonary function in infants with respiratory syncytial virus bronchiolitis]. Zhonghua Yi Xue Za Zhi. 2002 Feb 10;82(3):182-5. Chinese. PMID: 11953156.

25. Qi YY, Jiang GL, Wang LB, Wan CZ, Zhang XB, Qian LL. Lung Function in Wheezing Infants after Acute Lower Respiratory Tract Infection and Its Association with Respiratory Outcome. Chin Med J (Engl). 20175 th Jan 2017;130(1):4-10. doi: 10.4103/0366-6999.196577. PMID: 28051016; PMCID: PMC5221110.

26. Willson DF, Landrigan CP, Horn SD, Smout RJ. Complications in infants hospitalized for bronchiolitis or respiratory syncytial virus pneumonia. J Pediatr. 2003 Nov;143(5 Suppl):S142-9. doi: 10.1067/s0022-3476(03)00514-6. PMID: 14615713.

27. Caplow J, McBride SC, Steil GM, Wong J. Changes in cardiac output and stroke volume as measured by non-invasive CO monitoring in infants with RSV bronchiolitis. J Clin Monit Comput. 2012 Jun;26(3):197-205. doi: 10.1007/s10877012-9361-1. Epub 2012 Apr 24. PMID: 22526738.

28. Rodríguez-González M, Estepa-Pedregosa L, Estalella-Mendoza A, Castellano-Martínez A, Rodríguez-Campoy P, Carlos Flores-González J. Early elevated NT-proBNP but not troponin I is associated with severe bronchiolitis in infants. Clin Chim Acta. 2021 Jul;518:173-179. doi: 10.1016/j.cca.2021.03.023. Epub 2021 Apr 6. PMID: 33831420.

29. Rodriguez-Gonzalez M, Perez-Reviriego AA, Castellano-Martinez A, Lubian-Lopez S, Benavente-Fernandez I. Left Ventricular Dysfunction and Plasmatic NT-proBNP Are Associated with Adverse Evolution in Respiratory Syncytial Virus Bronchiolitis. Diagnostics (Basel). 2019 Jul 27;9(3):85. doi: 10.3390/diagnostics9030085. PMID: 31357664; PMCID: PMC6787702. 
30. Rodriguez-Gonzalez M, Benavente-Fernandez I, Castellano-Martinez A, Lechuga-Sancho AM, Lubian-Lopez SP. NTproBNP plasma levels as biomarkers for pulmonary hypertension in healthy infants with respiratory syncytial virus infection. Biomark Med. 2019 Jun;13(8):605-618. doi: 10.2217/bmm-2018-0348. Epub 2019 Jun 3. PMID: 31157543.

31. Thorburn K, Eisenhut M, Shauq A, Narayanswamy S, Burgess M. Right ventricular function in children with severe respiratory syncytial virus (RSV) bronchiolitis. Minerva Anestesiol. 2011 Jan;77(1):46-53. Epub 2010 Nov 24. PMID: 21102401.

32. Eisenhut M, Sidaras D, Johnson R, Newland P, Thorburn K. Cardiac Troponin T levels and myocardial involvement in children with severe respiratory syncytial virus lung disease. Acta Paediatr. 2004 Jul;93(7):887-90. PMID: 15303802.

33. Claudia Massolo A, Vanina Cantone G, Maria Caterina Musolino A, Corsini I, Patel N, Evangelisti M, Monaco F, Pia Villa M, Braguglia A. Myocardial strain on admission predicts disease severity in infants hospitalized for bronchiolitis. Pediatr Pulmonol. 2020 May;55(5):1217-1223. doi: 10.1002/ppul.24712. Epub 2020 Mar 5. PMID: 32134213.

34. Kimura D, Saravia J, Jaligama S, McNamara I, Vu LD, Sullivan RD, Mancarella S, You D, Cormier SA. New mouse model of pulmonary hypertension induced by respiratory syncytial virus bronchiolitis. Am J Physiol Heart Circ Physiol. 2018 Sep 1;315(3):H581-H589. doi: 10.1152/ajpheart.00627.2017. Epub 2018 Jun 15. PMID: 29906223; PMCID: PMC6172644.

35. Vu LD, Saravia J, Jaligama S, Baboeram Panday RV, Sullivan RD, Mancarella S, Cormier SA, Kimura D. Deficiency in ST2 signaling ameliorates RSV-associated pulmonary hypertension. Am J Physiol Heart Circ Physiol. 2021 Aug 1;321(2):H309-H317. doi: 10.1152/ajpheart.00018.2021. Epub 2021 Jun 25. PMID: 34170196; PMCID: PMC8410119.

36. Burkett DA, Slorach C, Patel SS, Redington AN, Ivy DD, Mertens L, Younoszai AK, Friedberg MK. Impact of Pulmonary Hemodynamics and Ventricular Interdependence on Left Ventricular Diastolic Function in Children With Pulmonary Hypertension. Circ Cardiovasc Imaging. 2016 Sep;9(9):10.1161/CIRCIMAGING.116.004612 e004612. doi: 10.1161/CIRCIMAGING.116.004612. PMID: 27581953; PMCID: PMC5012318.

37. Driessen MM, Hui W, Bijnens BH, Dragulescu A, Mertens L, Meijboom FJ, Friedberg MK. Adverse ventricularventricular interactions in right ventricular pressure load: Insights from pediatric pulmonary hypertension versus pulmonary stenosis. Physiol Rep. 2016 Jun;4(11):e12833. doi: 10.14814/phy2.12833. PMID: 27302992; PMCID: PMC4908502.

38. Mahmood SS, Pinsky MR. Heart-lung interactions during mechanical ventilation: the basics. Ann Transl Med. 2018 Sep;6(18):349. doi: 10.21037/atm.2018.04.29. PMID: 30370276; PMCID: PMC6186561.

39. Kimura D, McNamara IF, Wang J, Fowke JH, West AN, Philip R. Pulmonary hypertension during respiratory syncytial virus bronchiolitis: a risk factor for severity of illness. Cardiol Young. 2019 May;29(5):615-619. doi: 10.1017/S1047951119000313. Epub 2019 May 20. PMID: 31104634.

40. Habash S, Laser KT, Moosmann J, Reif R, Adler W, Glöckler M, Kececioglu D, Dittrich S. Normal values of the pulmonary artery acceleration time (PAAT) and the right ventricular ejection time (RVET) in children and adolescents and the impact of the PAAT/RVET-index in the assessment of pulmonary hypertension. Int J Cardiovasc Imaging. 2019 Feb;35(2):295-306. doi: 10.1007/s10554-019-01540-w. Epub 2019 Jan 28. PMID: 30689192.

41. Ord HL, Griksaitis MJ. Fifteen-minute consultation: Using point of care ultrasound to assess children with respiratory failure. Arch Dis Child Educ Pract Ed. 2019 Feb;104(1):2-10. doi: 10.1136/archdischild-2017-313795. Epub 2018 May 25. PMID: 29802097.

42. Musolino AM, Buonsenso D, Massolo AC, Gallo M, Supino MC, Boccuzzi E. Point of care ultrasound in the paediatric acute care setting: Getting to the 'heart' of respiratory distress. J Paediatr Child Health. 2021 Mar;57(3):318-322. doi: 10.1111/jpc.15308. Epub 2020 Dec 9. PMID: 33295654. 
